ВІСНИК

ОДЕСЬКОГО НАЦІОНАЛЬНОГО

МОРСЬКОГО УНІВЕРСИТЕТУ

№ 1 (64), 2021
HERALD

OF THE ODESSA NATIONAL

MARITIME UNIVERSITY № 1 (64), 2021

УДК 519.08

DOI 10.47049/2226-1893-2021-1-157-174

\title{
КОГНИТИВНЫЕ ТЕХНОЛОГИИ УПРАВЛЕНИЯ ЗНАНИЯМИ
}

\author{
Лб.С. Чернова \\ к.т.н., доцент, \\ доцент кафедры «Информационные управляющие системы и технологии»
}

Национальньй университет кораблестроения им. адмирала Макарова

Аннотация. В статье приводится определение когнитивной технологии, рассматриваются условия управления знаниями с помощью когнитивных технологий, включающих информационные технологии. Описаны основные технологии, предусматривающие управление знаниями, предоставлены отнотения технологий «управления знаниями» и «бизнес-интеллекта» в контексте интеллектуальности.

Рассмотрены когнитивные модели управления знаниями и дань инструменты для системы управления знаниями на основе выбора приоритетных организащионных знаниевых ресурсов, поддержсивающих актуальность, обновление и использование знаний в своей деятельности. В результате когнитивной структуризации происходит разработка неформального описания знаний, которые изображсаются в виде графа. На уровне когнитивной модели каждая связь рассматривается до соответствующего уравнения, которое может содержать как количественные, так и качественные переменные.

Исследованы когнитивные технологии в анализе, прогнозировании, принятии решений, управлении сложными системами, позволяющие применять методологию когнитивного моделирования к решению слабоструктурированных проблем различных сложных систем, которые в исследовании и разработке таких систем оказываются достаточно продуктивными. Рассмотрены когнитивные технологии, способные изменить подход в работе со знаниями, включающими рутинные операции. Установлено, что использование таких технологий улучшает качество работы в результате более эффективного использования знаний, сокращается время рабочих операчий, повышается результативность экспертной поддержки.

Ключевые слова: управление знаниями, когнитивные технологии, информационные технологии, когнитивное моделирование, функииональные графы.

(С) Чернова Лб.С., 2021 
ВІСНИК

ОДЕСЬКОГО НАЦІОНАЛЬНОГО

МОРСЬКОГО УНІВЕРСИТЕТУ

№ 1 (64), 2021
HERALD

OF THE ODESSA NATIONAL

MARITIME UNIVERSITY № 1 (64), 2021

УДК 519.08

DOI 10.47049/2226-1893-2021-1-157-174

\title{
КОГНІТИВНІ ТЕХНОЛОГІЇ УПРАВЛІННЯ ЗНАННЯМИ
}

\author{
Лб. С. Чернова \\ к.т.н., доцент, \\ доцент кафедри «Інформаційні управляючі системи та технології» \\ Національний університет кораблебудування ім. адмірала Макарова
}

Анотація. У статті наводиться визначення когнітивної технології, розглядаються умови управління знаннями за допомогою когнітивних технологій, щзо включають інформаційні технології. Описано основні технології, щзо передбачають управління знаннями, надані відносини технологій «управління знаннями» $i$ «бізнес-інтелекту» в контексті інтелектуальності.

Розглянуто когнітивні моделі управління знаннями і дані інструменти для системи управління знаннями на основі вибору пріоритетних організаційних знаннєвих ресурсів, що підтримують актуальність, оновлення та використання знань у своїй діяльності. У результаті когнітивної структуризачії відбувається розробка неформального опису знань, які зображуються у вигляді графа. На рівні когнітивної моделі кожен зв'язок розглядається до відповідного рівняння, яке може містити як кількісні, так і якісні змінні.

Досліджено когнітивні технологї в аналізі, прогнозуванні, прийнятті рішень, управлінні складними системами, щуо дозволяють застосовувати методологію когнітивного моделювання до вирішення слабоструктурованих проблем різних складних систем, які в дослідженні $i$ розробиі таких систем виявляються досить продуктивними. Розглянуто когнітивні технології, здатні змінити підхід в роботі зі знаннями, щзо включають рутинні операції. Встановлено, щзо використання таких технологій покрашує якість роботи в результаті більи ефективного використання знань, скорочується час робочих операцій, підвищується результативність експертної підтримки.

Ключові слова: управління знаннями, когнітивні технологї, технології, когнітивне моделювання, функціональні графи.

UDC 519.08

DOI 10.47049/2226-1893-2021-1-157-174

\section{COGNITIVE KNOWLEDGE MANAGEMENT TECHNOLOGIES}

\section{Lb. S. Chernova}

Ph.D., Associate Professor, Associate Professor of «Information Control Systems and Technologies» National University of Shipbuilding named after Admiral Makarov 
Abstract. The article provides a definition of cognitive technology, considers the conditions of knowledge management through cognitive technologies, including information technology. The main technologies involving knowledge management are described, the relations of technologies «know-ledge management» and «business intelligence» in the context of intelligence are given.

Cognitive models of knowledge management and given tools for the knowledge management system based on the choice of priority organizational knowledge resources that support the relevance, updating and use of knowledge in their activities. As a result of cognitive structuring, an informal description of knowledge is developed, which is represented in the form of a graph. At the level of the cognitive model, each relationship is considered to the corresponding equation, which may contain both quantitative and qualitative variables.

The use of cognitive technologies in the analysis, forecasting, decisionmaking, management of complex systems allows to apply the methodology of cognitive modeling to solve poorly structured problems of various complex systems.

The cognitive approach to research and development of such systems is quite productive. Cognitive technologies can change the approach to working with knowledge that includes routine operations. The use of such technologies improves the quality of work as a result of more efficient use of knowledge, reduces the time of use of work operations, increases the effectiveness of expert support.

Keywords: knowledge management, cognitive technologies, information technologies, cognitive modeling, functional graphs.

Вступление. В настоящее время опытные профессионалы, занимающееся управлением знаниями, стали неотъемлемой частью мира информационных технологий. Для того, чтобы преуспеть в глобальной информационной экономике, необходима интеллектуальная, исчерпывающая и простая в использовании система для управления запасами знаний, а также система доступа к знаниям и системам приобретения новых знаний.

С точки зрения целей общества, где доминируют информационные технологии, знания - это просто интеллект, используемый в работе. Знания, приобретенные фактическим опытом, продуктивны только тогда, когда они используются при выполнении работы или интегрируются в процесс выполнения работы. Знания приобретают разные формы и, поэтому ими становится сложнее управлять. Часто знания оказываются чемто большим, чем просто информацией и данными о событиях, продуктах или процедурах.

Управление знаниями - это распространение и поиск опыта людей и актуальной информации в среде связанных между собой людей или 
групп людей. При управлении информацией технология есть фокус сбора, распространения и многогранного использования информации.

Ключом к управлению знаниями является технология доставки знаний нужным людям в установленное время. Люди, процессы, технологии - строительные блоки успеха на сегодняшних рынках, переполненных информацией. Для поддержки человека при его работе над нововведениями и его стремлением к прогрессу, необходима технологическая инфраструктура, обеспечивающая условия для корпоративных знаний и быстрой практической выработки новых идей и решений.

Знание - это валюта, а совершенствование когнитивных технологий позволяет работать с уже имеющимися и полученными в процессе работы знаниями.

Задачи исследования. Выявление возможности использовать понятие «управление знаниями» как методологический ориентир для построения концепции разработки когнитивных технологий управления знаниями. Провести анализ исследования обобщенной модели управления знаниями. Определить рамки взаимодействия технологий «управления» знаниями и «бизнес-интеллекта».

Анализ литературы. Проведя анализ достижений ученых в сфере управления знаниями $[1 ; 2 ; 3 ; 4 ; 5 ; 6 ; 7 ; 8]$ установлено, что в методологии управления проектами недостаточно изучен компонент, связанный с когнитивными технологиями управления знаниями.

Изложение основного материала. Деятельность (активность) любой системы в первую очередь определяется совокупностью технологий, которые она реализует как интегрированная и открытая система. Технологий, как способов и средств, которые и получают и применяют знания. Таких технологий, как производственные, организационные, политические, информационные, когнитивные и иные способы и средства активности систем. Когнитивные технологии - это технологии изучения процесса познания (мышления) и технологии применения знаний об этом процессе [9]. Методологическую основу технологий в значительной степени обеспечивает возможность обмена формальными знаниями.

Современные технологии обеспечения управления позволяют в принципе управлять любыми сложными детерминированными системами (и любой формализуемой информацией), решать системы уравнений любой сложности и оперировать прочими сложностями. Поэтому сегодня сложность управления определяется возможностью управления такими технологиями активности системы, которые являются не полностью определенными.

В настоящее время быстро развивается целый ряд новых технологий планирования, принятия решений и обучения. Особенностью многих из этих технологий является использование технологии управления знаниями. 
Когнитивные технологии - способы и алгоритмы достижения целей субъектов, опирающееся на данные о процессах познания, обучения, коммуникации, обработки информации, компьютерные информационные технологии, математическое моделирование элементов сознания, ряд других научных направлений, относящихся к сфере фундаментальной науки. Именно в таком смысле надо понимать когнитивные технологии [10].

Управление знаниями (Knowledge Management, KM) осуществляется с помощью когнитивных технологий, включающих информационные технологии. В Википедии дано следующее определение управления знаниями (КМ) - это методология, направленная на повышение уровня конкурентоспособности и защищенности компании за счет использования полного набора инструментов охраны, управления и экономики нематериальных активов компании. Рассматривает стратегии, направленные на предоставление вовремя нужных знаний тем членам сообщества, которым эти знания необходимы для того, чтобы повысить эффективность деятельности сообщества.

Формализация знаний на бумажных носителях практически полностью ушла в прошлое, так как она менее удобна и надежна, чем электронный вид. Информационные технологии позволяют обеспечить сохранность знаний в организации и эффективность операций с ними. Развитие систем обработки данных и искусственного интеллекта, а также распространение технологий big data играет ключевую роль в управлении знаниями в организации в современной среде, переполненной информацией.

В число основных технологий, поддерживающих КМ, входят (рис. 1):

- добыча данных и текстов - распознавание образов, выделение значимых закономерностей из данных, находящихся в хранилищах или входных или выходных потоках. Эти методы основываются на статистическом моделировании, нейронных сетях, генетических алгоритмах и др.;

- системы управления документооборотом - хранение, архивирование, индексирование, разметка и публикация документов;

- средства для организации совместной работы - сети internet, технологии групповой работы, синхронные и асинхронные конфигурации;

- корпоративные порталы знаний;

- средства, поддерживающие принятие решений - экспертные системы, системы, поддерживающие дискуссионные группы и т.д.

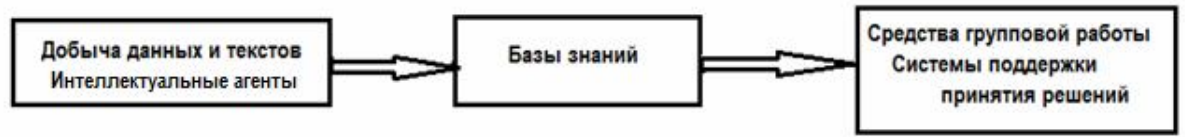

Рис.1. Технологии, поддерживающие управление знаниями 
По отношению к базам знаний, которые являются центральной частью (рис. 1), технологии можно разделить на входные и выходные. Сгруппированные по принципу «кто с кем взаимодействует», эти технологии, а также более традиционные современные технологии передачи знаний приведены в табл. 1.

Таблий 1

\section{Технологии КM}

\begin{tabular}{|l|l|l|}
\hline & \multicolumn{1}{|c|}{ Работа с информацией } & \multicolumn{1}{c|}{ Работа со знаниями } \\
\hline $\begin{array}{l}\text { Человек - } \\
\text { Человек }\end{array}$ & $\begin{array}{l}\text { Электронная почта, теле- } \\
\text { конференции }\end{array}$ & $\begin{array}{l}\text { Системы телеконферен- } \\
\text { ций, видеоконференции }\end{array}$ \\
\hline $\begin{array}{l}\text { Человек - } \\
\text { Компьютер }\end{array}$ & $\begin{array}{l}\text { Системы управления доку- } \\
\text { ментооборотом, базы данных } \\
\text { и средства доступа к ним }\end{array}$ & $\begin{array}{l}\text { Экспертные системы, } \\
\text { системы поддержки } \\
\text { принятия решений }\end{array}$ \\
\hline $\begin{array}{l}\text { Компьютер - } \\
\text { Компьютер }\end{array}$ & $\begin{array}{l}\text { Нейронные сети, интел- } \\
\text { дектуальные агенты }\end{array}$ \\
\hline
\end{tabular}

В случае когнитивных технологий наука находится в начале пути. В этой области существует огромный потенциал развития.

Мир вступает в полосу быстрых изменений в начавшийся период кризиса, который, вероятно, займет не одно десятилетие. Поэтому практическая потребность в росте темпов принятия управленческих решений очевидна. Естественно, системы оперативного управления не должны отставать от тех изменений, реакцией на которые должны быть своевременно принимаемые решения. И без развитой системы когнитивных технологий тут не обойтись.

В эпоху научно-технического оптимизма считалось, что развитие должно происходить по закону геометрической прогрессии - в одинаковое число раз за одинаковые промежутки времени, см. рис. 2 (или на языке дифференциальных уравнений по экспоненте $x=a x$, где $x$-интеграционный показатель, характеризующий отрасль, $a-$ коэффициент, часто называемый мальтузианским). Однако реальность науки и техники показывает, что отрасли и технологии характеризуются логическим законом $(x=a x(\bar{x}-x))$, где $\bar{x}-$ предельный уровень развития. При этом происходит насыщение, на которое выходят «старые» отрасли. Та же закономерность характерна для научных школ, направлений, целых областей исследований.

Знания, в свою очередь, стали основным объектом вложений вместо капитальных средств. Так, Япония инвестирует в заводы и оборудование $8 \%$ своего годового ВВП. При этом Япония вкладывает, как минимум, в 2 раза большую сумму в образование [11]. 


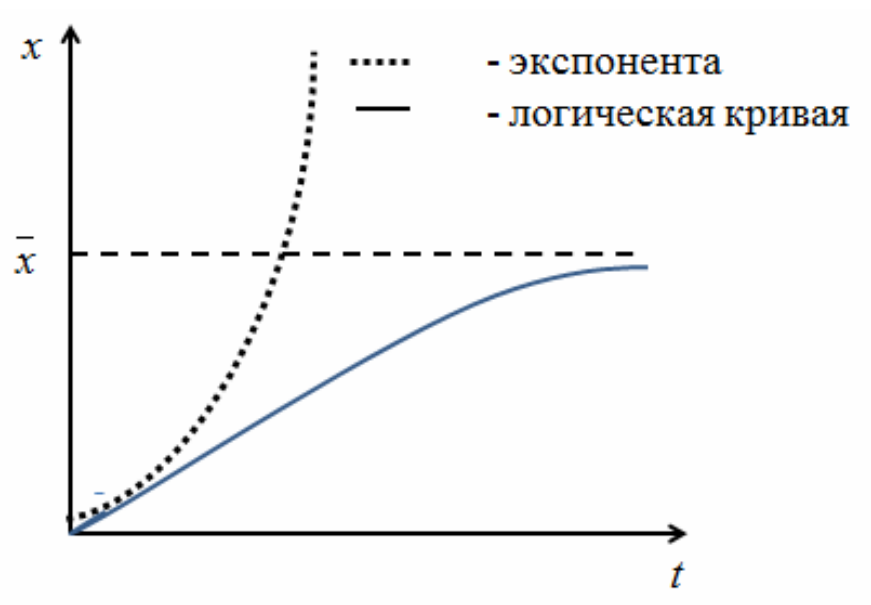

Рис. 2. Сравнение экспоненциального и линейного роста

В настоящее время в публикациях по компьютерным технологиям встречается совместно с термином «Управление знаниями» (КМ) «Бизнес-интеллект» (business intelligence - BI).

ВI в широком смысле слова определяет [12]:

- процесс превращения данных в информацию и знания о бизнесе для поддержки принятия улучшенных и неформальных решений;

- информационные технологии (методы и средства) сбора данных, консолидации информации и обеспечение доступа бизнес-пользователей к знаниям;

- знания о бизнесе, добытые в результате углубленного анализа детальных данных и консолидированной информации.

Технологии КМ и ВІ, имея общие методы и средства, дополняют друг друга [13].

На рис. 3 представлены две взаимосвязанные треугольные структуры «I-AI-BI» и « KM-KB-K». Первая - связана «интеллектом», вторая со «знанием». Лучше говорить о некотором «гексагоне интеллектуальности ВІ и КМ, т.к. все компоненты в этом шестиугольнике тесно связаны. Заметим, понятия интеллекта (Intelligence - I), искусственного интеллекта (Artificial intelligence - AI), знаний (Knowledge - K), баз знаний (Knowledge Base - KB) были введены давно, с общепризнанной недоопределенностью (видимо «навсегда»). 


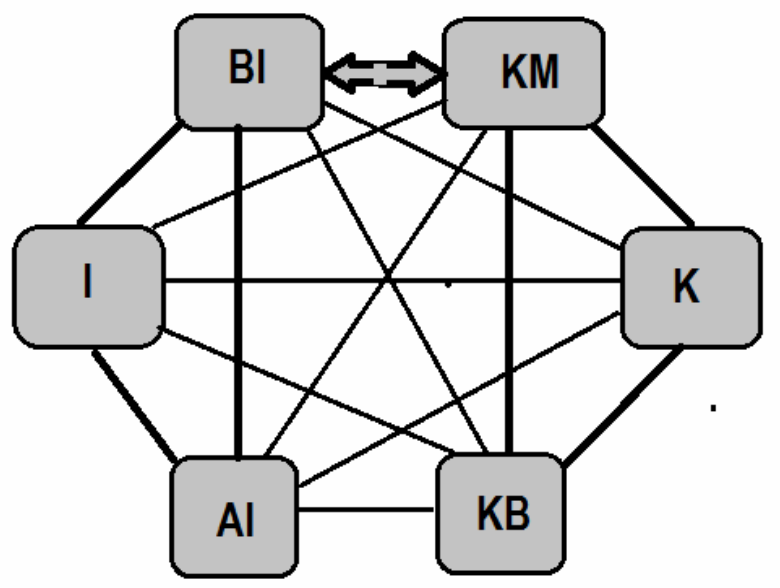

\section{Рис. 3. Отночения ВI-КМ в контексте «интеллектуальности»}

Первое определение гласило: «ВI - знания, добытые в бизнесе с использованием различных аппаратно-программных технологий. Такие технологии дают возможность организациям превращать данные в информацию, а затем информацию в знания», это определение четко разграничивает понятия «данные-информация-знания».

Данные, при этом, понимаются как реальность, которую компьютер записывает, хранит и обрабатывает - это «сырые данные».

Информация - это то, что человек в состоянии понять о реальности, а знания - это то, что в бизнесе используется для принятия решений.

В [14] предложена триада «данные-информация-знания», а в [15] эта структура детально анализировалась в приложении к информационным технологиям.

В процессе организации информации для получения знания часто применяют хранилища данных, а для представления этого знания пользователям - инструменты бизнес-интелекта. Разработаны программные приложения, которые обеспечивают пользователей возможностью проводить такой процесс для ответа на вопросы бизнеса и для выявления значимых тенденций или шаблонов в исследуемой информации. «Управление знаниями» - дисциплина более универсальная, отличающаяся более широкой полосой охвата. Она основана на интегральном подходе к сознанию, накоплению, и, в некотором смысле, управлению знаниями, хранящимся в виде документов различного рода, а также знаниями, принадлежащими сотрудникам предприятия. Поначалу КМ никак не было связано с информационными технологиями. Первоначальной целью было создание руководств и методик для оптимального использования интел- 
лектуального потенциала работников компаний. Знания были признаны экономической категорией, и спустя короткое время появились информационные технологии для работы с ними, а теперь и интеллектуальные.

Благодаря когнитивным технологиям эффективность обучения и использования знаний может вырости на порядок... Очевидно, страна, которая добьется этого, станет лидером завтрашнего дня, поэтому «гонка когнитивных технологий» уже началась.

В результате когнитивной структуризации происходит разработка неформального описания знаний о предметной области, которую можно наглядно изобразить в виде схемы, графа, матрицы, а также таблицы или текста. Наиболее удобной формой является граф - когнитивная карта (1).

Понятие когнитивной карты - карты познания - является исходным в когнитивном анализе и моделировании сложных ситуаций. Когнитивные карты являются схемами причинно-следственных связей, интерпретирующих мнения и взгляды лица принимающего решения.

С формальных позиций когнитивная карта - это знаковый ориентированный граф (орграф)

$$
G=<\mathrm{V}, \mathrm{E}>\text {, }
$$

где $V-$ множество вершин (объектов, концептов), вершины $V_{i} \hat{I} V, i=1,2, \ldots, k$ являются элементами изучаемой системы;

$E$ - множество дуг, дуги $e_{i j} \hat{I} E, i, j=1,2, \ldots, N \quad$ отражают взаимосвязь между вершинами $V_{i}$ и $V_{j}$; влияние $V_{i}$ на $V_{j}$; в изучаемой ситуации может быть положительным, когда увеличение (уменьшение) одного фактора приводит к увеличению (уменьшению) другого, отрицательным, когда увеличение (уменьшение) одного фактора приводит к уменьшению (увеличению) другого, или отсутствовать (0) в рассматриваемый момент времени.

Матрица отношений когнитивной карты $A_{G}$ - это квадратная матрица, строки и столбцы которой помечены вершинами графа $G$, а на пересечении $i$-строки, $j$-столбца стоят (или нет) единицы, если существует (не существует) отношение между элементами $V_{i}$ и $V_{j}$, т.е.

$$
A_{G}=\left[a_{i j}\right]_{k^{\prime} k}, a_{i j}=\left\{\begin{array}{l}
1, \text { если } V_{i} \text { связано } V_{j} \\
0, \text { если в противном случае }
\end{array}\right\}
$$

Отношение $a_{i j}$ может принимать значение «+1» или «-1».

Отношение между переменными (взаимодействие факторов) - это количественное или качественное описание влияния изменения одной переменной на другие. 
На уровне когнитивной модели каждая связь между факторами когнитивной карты раскрывается до соответствующего уравнения, которое может содержать как количественные (измеряемые) переменные, так и качественные (неизменяемые) переменные. Количественные переменные входят естественным образом в виде их численных значений. Каждой качественной переменной может быть поставлена в соответствие совокупность лингвистических переменных типа «сильно», «умеренно», «слабо» и т.п., которым соответствует числовая шкала [0;1], на которой задается функция принадлежности. По мере накопления знаний о процессах становится возможным более детально раскрывать характер связей между факторами (процедуры «data mining»). Все это порождает различные типы когнитивных моделей.

Основные типы когнитивных моделей:

- векторный функциональный граф (частными случаями которого являются знаковый орграф, взвешенный знаковый орграф и простейший функциональный граф);

- параметрический векторный функциональный граф;

- модифицированный граф.

Векторный функциональный граф (Ф-граф) - это кортеж

$$
F=<G, X, F>\text {, }
$$

где

1) $G=<V, E>-$ ориентированный граф;

2) $X$ - множество параметров вершин $V$;

$$
X=\left\{X^{\left(V_{i}\right)}\right\}, i=1,2, \ldots, k, X^{\left(V_{i}\right)}=\left\{x^{(i)} g\right\}, g=1,2, \ldots n_{i} ;
$$

т.е. каждой вершине ставится в соответствие вектор независимых друг от друга параметров $X^{\left(V_{i}\right)}$, (или один параметр $V_{j}$, если $\left.g=1\right) ; X: V \circledR R, R-$ множество вещественных чисел;

3) $F=F(X, E)=F\left(x_{i}, x_{j}, e_{i j}\right)$ - функционал преобразования дуг, ставящий в соответствии каждой дуге либо знак («+», «-»), либо весовой коэффициент $w_{i j}$, либо функцию $f\left(x_{i}, x_{j}, e_{i j}\right)=f_{i j}$. В зависимости от $F(X, E)$ вводится расширенное понятие орграфа.

Знаковый орграф (когнитивная карта) - это $F$ - граф, в котором

$$
F(X, E)=F\left(x_{i}, x_{j}, e_{i j}\right)=\left\{\begin{array}{c}
+1, \text { если рост (падение) } \mathrm{x}_{i} \text { влечет } \\
\text { за собой рост (падение) } \mathrm{x}_{j} ; \\
-1, \text { если рост (падение) } \mathrm{x}_{i} \text { влечет } \\
\text { за собой падение (рост) } \mathrm{x}_{j} .
\end{array}\right.
$$


Взвешенный знаковый орграф - это F-граф, в котором

$$
F(X, E)=F\left(x_{i}, x_{j}, e_{i j}\right)=\left\{\begin{array}{c}
+\omega_{i j}, \text { если рост (падение) } \mathrm{x}_{i} \text { влечет } \\
\text { за собой рост (падение) } \mathrm{x}_{j} ; \\
-\omega_{i j}, \text { если рост (падение) } \mathrm{x}_{i} \text { влечет } \\
\text { за собой падение (рост) } \mathrm{x}_{j} .
\end{array}\right.
$$

где $w_{i j}$ - весовой коэффициент; $w_{i j} \hat{I} W, W$ - множество всех дуг, $W: E^{\prime} X ® R . R$ - множество вещественных чисел. Оценка $w_{i j}$ может определяться одним числом или быть интервальной.

Простейтий функциональнылй граф - это Ф-граф, в котором

$$
F(X, E)=F\left(x_{i}, x_{j}, e_{i j}\right)=\left\{\begin{array}{c}
+f_{i j}, \text { если рост (падение) } \mathrm{x}_{i} \text { влечет } \\
\text { за собой рост (падение) } \mathrm{x}_{j} ; \\
-f_{i j}, \text { если рост (падение) } \mathrm{x}_{i} \text { влечет } \\
\text { за собой падение (рост) } \mathrm{x}_{j} .
\end{array}\right.
$$

$f_{i j}$ - это функциональная зависимость параметров вершин, которая ставится в соответствии каждой дуге. Зависимость $f_{i j}$ может быть не только функциональной, но и стохастической $h_{i j}$.

Определение Ф-графов может быть обобщено нижеследующим образом (согласно Ф.С. Робертсу).

Параметрический векторный функииональный граф $\Phi_{n}-$ это кортеж

$$
\Phi_{n}<V, E>, X, F, q>
$$

в котором

1) $G=\left\langle V, E>, V=\left\{v_{i} \mid v_{i} \hat{I} V, i=1,2, \ldots, k\right\} ; E=\left\{e_{i} \mid e_{i} \hat{I} E, i=1,2, \ldots, k\right\}\right.$;

$G$ - ориентированный граф;

2) $X: V \circledast q, X-$ множество параметров вершин,

$$
X=\{\mid \hat{I} X, i=1,2, \ldots, k\},=\left\{x^{(i)} g\right\},
$$

$g=1,2, \ldots, l . x^{(i)} g-g-$ параметр вершины $i, j=1,2, \ldots k$

если $g=1, \operatorname{mox}^{(i)} g=x_{i}$;

$q$ - пространство параметров вершин;

3) $F=F(X, E)$ - функционал преобразования дуг, $F: E^{\prime} X^{\prime} q^{\circledR} R$.

Определение параметров характеристики $f_{i j}$ включает определение шкалы, показателей, метода, точности, единицы измерения. 
Для отражения динамики происходящих в системе под воздействием всевозможных возмущений изменений, в модель вводится время.

Непрерывное временное пространство $T$ - пространство, в котором происходят динамические процессы.

Последовательность моментов времени $\left\{t_{n}\right\}$ - это моменты, выделенные в пространстве $T$ по определенным правилам и для которых определены воздействия на систему и правила изменения состояний системы.

Полагаем, что изменения состояний системы происходят мгновенно.

Рассмотрим непрерывный и дискретный случаи наступления очередного момента времени в последовательности $T_{n}$.

В дискретном случае $t \hat{I}\left(t_{n}, t_{n+1}\right)$, причем существуют пределы для переменных состояния и «разрывное» изменение состояния может происходить только в моменты $\left\{t_{n}\right\}$, эти состояния обозначим соответственно $S\left(t_{-}\right)$u $S\left(t_{+}\right)$, т.е. до и после $t_{n}$.

$B$ непрерывном случае состояние системы считается неизменным на всем интервале $t \hat{I}\left(t_{n}, t_{n+1}\right)$. В непрерывном случае последовательность $\left\{t_{n}\right\}$ является следствием дискретизации временного пространства, состояния $S\left(t_{n}\right)$ системы на интервале $\left(t_{n}, t_{n+1}\right)$ интерполируются по заранее заданной схеме.

Продвижение системы во времени от одного состояния к другому требует правила построения последовательностей $<t_{n}, S_{n}, Q_{n}>$, где $S_{n}==S\left(t_{n}\right)$ - состояние системы, $Q_{n}$ - внешнее воздействие в момент времени $t_{n}, n=1,2, \ldots$.

Для моделирования взаимодействия сложных по природе процессов требуется определить схему взаимовлияния факторов и построение механизмов реакции на возмущение и его передачу. Введем понятия: элементарное возмущение (элементарный импульс), поступившее возмущение, генерируемое возмущение, которые позволят ввести далее определение модифицированного МФ-графа, отражающего динамику процессов в системе.

Рассмотрим ряд определений.

Импульс (Ітр) (возмущение) $P_{v}(t)$ в вершине $v \hat{I} V$ в момент времени $P t \hat{I} T_{v}(t)$ - это изменение параметра в этой вершине в момент времени $t: P_{v}(t)=X_{v}\left(t_{+}\right)-X_{v}\left(t_{-}\right)$. 
Внешний импульс в момент $t$ - это совокупность

$$
Q(t)=\left\{Q_{v}(t), v \hat{I} V\right\} .
$$

Для выделения двух самостоятельных механизмов в системе механизма реакиии на поступивщее возмущение и механизма генерации возмущения - произведем разделение понятия импульса в вершине на три взаимосвязанных понятия: элементарное, поступившее и генерируемое возмущения.

Элементарное возмущение - это импульс, несущий информацию о воздействии одной вершины на другую. В совокупности элементарных импульсов выделяются внутренний и внешний.

Поступивщее возмущение $\left(P_{x}\right)$ - это совокупность элементарных импульсов, воздействующих на вершину в один и тот же момент времени.

Генерируемое возмущение $\left(P_{u v}\right)$ - возмущение, формируемое в вершине для воздействия на другие вершины через дуги.

Модифицированный МФ-граф - это кортеж

$$
M \Phi=<G,\left(X, D p, P_{x v}, P_{u v}, m, n, D_{u}, P_{e}\right),\left(h, W, D_{b}\right), e>,
$$

в котором $G=<V, E>-$ ориентированный граф (когнитивная карта);

$\left(X, D p, P_{x v}, P_{u v}, m, n, D_{u}, P_{e}\right)$ - параметрические характеристики вершин и механизмы преобразования возмущений.

Объект моделирования можно рассматривать как совокупность взаимодействующих между собой динамических процессов, протекающих в реальном времени. В модели процессов также должно присутствовать время, но при моделировании разными типами графов это время может не иметь смысла времени, а отражать только последовательность изменений состояний. Это имеет место для знаковых орграфов и знаковых взвешенных орграфов.

Построение модели с использованием МФ-графов требует определения:

- совокупности факторов моделируемого объекта, которые представляются множеством вершин $V$ графа $G$;

- совокупности воздействия факторов друг на друга (отношений между факторами), которые представляются множеством дуг $E$ графа G;

- характеристик дуг, которые представляются кортежем $\left(h, W, D_{b}\right)$;

- внешних возмущений, представляемых подмножеством импульсов $Q I ́\{\operatorname{Imp}\}^{*}$;

- предельной точности вычислений $e$.

Заметим, что в немодифицированном Ф-графе генерируемое возмущение совпадает с поступившим возмущением и равняется сумме эле- 
ментарных импульсов, воздействующих на вершину в данный момент времени.

Под влиянием различных возмущений, значения переменных в вершинах графа могут изменяться; сигнал, поступивший в одну из вершин, распространяется по цепочке на остальные, усиливаясь или затухая.

Правило (PR) изменения параметров в вериинах в момент $t_{n+1}$

Пусть параметр $x_{i}$ зависит от времени, т.е. $x_{i}(t), t=1,2,3, \ldots$ Тогда можно определить процесс распространения возмущения по графу, т.е. переход системы из состояния $t-1$ в $t, t+1, \ldots$.

Пусть значение $x_{i}(t+1)$ в вершине $v_{i}$ зависит от $x_{i}(t)$ и от вершин, смежных с $V_{i}$. Пусть $V_{i}$ смежная с $V_{j}$ пусть $p_{j}(t)$-изменение в вершине $V_{j}$ в момент времени $t$, тогда влияние этого изменения на параметр $x_{i}$ в момент $t$ будет описываться функцией $\pm p_{j}(t)$ в зависимости от знака дуги, соединяющего $V_{i}$ и $V_{j}$.

В общем случае, если имеется несколько вершин $V_{j}$, смежных с $V_{i}$, процесс распространения возмущения по графу определяется правилом

$$
x_{i}(t+1)=x_{i}(t)+\sum_{j=1}^{k-1} f\left(x_{i}, x_{j}, e_{i j}\right) p_{j}(t)
$$

при известных начальных значениях $X(0)$ во всех вершинах и начальном векторе возмущения $P(0)$.

Моделирование можно проводить шагами или импульсами. Суть такого моделирования состоит в том, что в одной из вершин графа задается определенное изменение. Эта вершина актуализирует всю систему показателей, т.е. связанных с ней вершин, в большей или меньшей степени. Таких вершин может быть несколько, их принято называть активизирующими.

Представим функцию $f_{i j}$ между вершинами $V_{j}, V_{i}$ с помощью коэффициентов $j i, w_{j i}$, характеризующих знак $\left(l_{j i}:\langle+»)\right.$ или «-») и степень влияния $w_{j i}$ параметра вершины $V_{j}$ на параметр вершины $V_{i}$; функцию $p_{j}(t)$ влияния изменения в смежной с $V_{j}$ вершине $V_{i}$ заменим импульсом $p(n)=x(n+1)-x(n)$, где $x(n), x(n+1)-$ величины показателя в вершине $V$ при шагах имитации в момент $t=n$ и следующим за ним $t=n+1$. Тогда формула (9) преобразуется к виду 


$$
x_{i}(n+1)=x_{i}(n)+\sum_{j=1}^{k-1} \lambda_{j i} \omega_{j i}\left[x_{j}(n)-x_{j}(n)\right] .
$$

В моделях этого типа коэффициенты $\omega_{i j}$, характеризующие взаимовлияние смежных вершин, могут определяться либо экспертно, либо статистическими методами.

В такие модели могут вводиться еще и лаги, т.е. задержки передачи воздействия по каждой дуге во времени.

Правило (PR) изменения параметров в вершинах в момент $t=n+1$, если в момент времени $t_{n}$ в вершины поступили импульсы

$$
x_{i}\left(t_{n+1}\right)=x_{i}\left(t_{n}\right)+\sum_{v_{j} e=e_{i j} \varepsilon E}^{k-1} f\left(x_{i}, x_{j}, e_{i j}\right) P_{j}\left(t_{n}\right)+Q_{i}\left(t_{n+1}\right) .
$$

Импульс, порожденный изменением параметра в вершине

$$
P_{i}\left(t_{n+1}\right)=\sum_{v_{j} e=e_{i j} \varepsilon E}^{k-1} f\left(x_{i}, x_{j}, e_{i j}\right) P_{j}\left(t_{n}\right)+Q_{i}\left(t_{n+1}\right) .
$$

Так как в Ф-графе импульс в импульсном процессе представляется упорядоченной последовательностью без привязки ко времени, то можно использовать запись формул «в $n$-й момент времени». Тогда

$$
x_{i}(n+1)=x_{i}(n)+\sum_{v_{j} e=e_{i j} \varepsilon E}^{k-1} f\left(x_{i}, x_{j}, e_{i j}\right) P_{j}(n)+Q_{i}(n+1) .
$$

При этом последовательность $<n, X\left(t_{n}\right), Q\left(t_{n}\right)>$ является модельным представлением системы $<t_{n}, S_{n}, B_{n}>$.

Математическая модель импульсных прочессов в матричном виде (на знаковых графах).

Пусть $Q_{t}=\left\{q_{i t}\right\}_{i=1}^{k}, t=0,1,2, \ldots-$ вектор внешних импульсов $q_{i t}$, вносимых в вершины $v_{i}$ в момент времени $t$;

$$
X_{t}=\left\{x_{i t}\right\}_{i=1}^{k}, t=0,1,2, \ldots-\text { вектор значений параметров } x_{i t} \text { вер- }
$$

шин $v_{i}$ в момент времени $t$;

$$
R_{t}=\left\{\Delta_{i t}\right\}_{i=1}^{k}-\text { вектор параметров вершин в момент времени } t \text {, }
$$
который задается уравнением

$$
R_{t}=X_{t}-X_{t-1}, t=1,2,3, \ldots
$$

Изменения параметров вершин задаются следующим уравнением:

$$
X_{t}=X_{t-1}+A R_{t-1}+Q_{t-1} .
$$

Получим из последнего уравнения выражение для $R_{t}$

$$
A^{t-1} Q_{0}+\ldots+A Q_{t-2}+I Q_{t-1},
$$

где $I$ - единичная матрица. 
Для частного случая импульсных процессов на когнитивных картах, называемых автономными (внешние импульсы вносятся только один раз в начале моделирования), $p_{i t}=0$, " $t^{3} 1$ и для изменения параметров получаем $\Delta_{l}^{t}=\sum_{i=1}^{k} a_{l i}^{t-1} p_{i o}, l=1,2 \ldots N ; a_{l i}^{f}$ - элемент матрицы $A^{t}=\left\{a_{l i}^{t}\right\}$.

Простейшим вариантом распространения возмущения является случай, когда $P(0)$ имеет лишь один ненулевой вход, т.е. возмущение поступает только в одну вершину $V_{i}$.Такие процессы принято называть простыми процессами распространения возмущений.

Заключение. Исследованы когнитивные методологии управления знаниями, поддерживающие синтез, анализ новых знаний и их использование, что позволяет развивать методологии управления проектами с встроенными когнитивными механизмами управления знаниями.

\section{ЛІТЕРАТУРА}

1. Мариничева.Управление знаниями на $100 \%$. Путеводитель для практикантов. - М.: Альпина Бизнес Букс. - 2008. 320 c.

2. Коулоулос Т.Т., Фраппаоло. Управление знаниями / Пер. с англ. - M., 2008. - $224 c$.

3. Тесля Ю.М., Хлевна Ю.Л., Егорченкова Н.Ю. Управління знаннями в мета-методології управління проектами. Управління проектами та розвиток виробництва: 3б. наук. праць. - Луганськ: Вид-во СНУ ім. В.Даля, 2016. -№ 4 (60). C.53-61.

4. Бушуев С.Д., Бушуева Н.С. Методология управления проектами как универсальная модель знаний. Управління проектами та розвиток виробництва: 3б. наук. праць. - Луганськ: Вид-во СНУ ім. В.Даля, 2003. - № 3 (8). - C.5-12.

5. Бабаев И.А. Инструменты моделирования знаний в управлении проектами. Управління проектами та розвиток виробництва: Зб. наук. праџь. - Луганськ: Вид-во СНУ ім. В. Даля, 2004. - № 2 (10). - C.10-24.

6. Глубокова Е.Н. Управление знаниями как основа построения образовательного процесса в современном университете // Известия Российского государственного педагогического университета им. А.И. Гериеена. - СПб., 2009. - № 100. C.48-56.

7. Силов В.Б. Принятие стратегических решений в нечеткой обстановке: Монография. -М.: ИНПРО-РЕС,1995. - 228 с. 
ВІСНИК

ОДЕСЬКОГО НАЦІОНАЛЬНОГО

МОРСЬКОГО УНІВЕРСИТЕТУ

№ 1 (64), 2021
HERALD

OF THE ODESSA NATIONAL

MARITIME UNIVERSITY

8. Смирнова Е.Е., Надеждин Е.Н. Нечеткая когнитивная модель механизма формирования профессиональных компетенций будущего бакалавра экономики // Информащия образования и науки. - 2016. - № 1(29). -175-184.

9. Малинеикий Г.Г., Маненков С.К., Митин Н.А., Шишов В.В. Когнитивный вызов и информачионные технологии // Экономические стратегии. - 2011. - № 9.

10. Малинеикий Г.Г. Когнитивный вызов и информационные технологии. Препринты НПМ, 2010. - № 46. -28 c.

11. Волков О.Г. Требования работодателей и государственный стандарт профессионального образования: Методическое пособие. - Чебоксары, 2009.

12. Артемьев В. Что такое Business Intelligence // Открытые системы. - 2003. - № 1.

13. Вальман Ю.Р., Вальман Р.Ю., Исматилова Л.Р., Яковенко Н.И. Бизнес-интеллект управления знаниями: понятия, средства, отношения.

14. Вальман Ю.Р., Скурихин В.И. Данные, информачия, знания в проектировании сложных объектов: трансформация и жизненные циклы // Сб. докладов 5-й Международной конференичи «Автоматизаџия и конструирането на технологични проиеси в машиностроенето». - Пловдия, Болгария, 1988.

15. Скурихин В.И., Квичев В.Г., Вальман Ю.Р., Яковенко Л.П. Информачионные технологии в испытаниях сложных объектов: методы и средства. - К.: Наукова думка, 1990. - 320 с.

\section{REFERENCES}

1. Marinicheva. Knowledge management by $100 \%$. Guide for trainees. - M.: Alpina Business Books, 2008. - 320 p.

2. Couloulos, T.T., Frappaolo. Knowledge management/Per. with English. Moscow, 2008. 224 p.

3. Tesla, Yu.M., Khlevna, Yu.L., Egorchenkova, N.Yu. Knowledge management in project management meta-methodology. Project management and production development: Coll. Science. works Lugansk: published by SNU. V. Dalya, 2016. № 4 (60). P.53-61.

4. Bushuev, S.D., Bushueva, N.S. Project management methodology as a universal model of knowledge. Project management and production development: Coll. Science. works Lugansk: published by SNU. V. Dalya, 2003. № 3 (8). P.5-12.

5. Babaev, I.A. Knowledge modeling tools in project management. Project management and production development: Coll. Science. works Lugansk: published by SNU. V. Dalia, 2004. № 2 (10). P.10-24. 
6. Glubokova, E.N. Knowledge management as a basis for building an educational process in a modern university / E.N. Glubokova // Izvestiya Rossiiskogo gosudarstvennogo pedagogicheskogo universiteta im. AI Herzen / SPb. 2009. № 100. P.48-56.

7. Silov, V.B. Making strategic decisions in a vague situation: a monograph. M.: INPRO-RES, 1995. 228 p.

8. Smirnova, E.E., Nadezhdin, E.N. Fuzzy cognitive model of the mechanism of formation of professional competencies of the future bachelor of economics // Information of education and science. 2016. № 1 (29). 175-184.

9. Malinetsky, G.G., Manenkov, S.K., Mitin, N.A., Shishov, V.V. Cognitive challenge and information technology // Economic strategies. 2011. № 9

10. Malinetsky, G.G. Cognitive challenge and information technology. NPM preprints, 2010. № 46. $28 \mathrm{p}$.

11. Volkov, O.G. Requirements of employers and the state standard of professional education. Methodical manual. Cheboksary, 2009

12. Artemyev, V. What is Business Intelligence // Open Systems. 2003. № 1 .

13. Valman, Y.R., Valman, R.Y., Ismatilova, L.R., Yakovenko, N.I. Business intelligence knowledge management: concepts, tools, relationships.

14. Valman, Y.R., Skurikhin, V.I. Data, information, knowledge in the design of complex objects: transformation and life cycles // Sb. reports of the 5th International Conference "Automation and design of technological processes in mechanical engineering». Plovdiv, Bulgaria. 1988.

15. Skurikhin, V.I., Kvichev, V.G., Valman, Y.R., Yakovenko, L.P. Information technologies in tests of complex objects: methods and means. Scientific opinion. Kiev, 1990. 320 p.

Стаття надійшла до редакиії 29.12.2020

Посилання на статтю: Чернова Лб.С. Когнитивные технологии управления знаниями // Вісник Одеського національного морського університету: 3б. наук. праць, 2021. № 1 (64). C. 157-174. DOI 10.47049/ 2226-1893-2021-1157-174.

Article received 29.12.2020

Reference a JournalArtic: Chernova Lb.S. Cognitive knowledge management technologies // Herald of the Odessa National Maritime University. 2021. 1(64), 157-174. DOI 10.47049/ 2226-1893-2021-1-157-174. 